Optimal Tuner Selection for Kalman Filter-Based Aircraft Engine Performance Estimation

Donald L. Simon and Sanjay Garg

Glenn Research Center, Cleveland, Ohio 


\section{NASA STI Program . . . in Profile}

Since its founding, NASA has been dedicated to the advancement of aeronautics and space science. The NASA Scientific and Technical Information (STI) program plays a key part in helping NASA maintain this important role.

The NASA STI Program operates under the auspices of the Agency Chief Information Officer. It collects, organizes, provides for archiving, and disseminates NASA's STI. The NASA STI program provides access to the NASA Aeronautics and Space Database and its public interface, the NASA Technical Reports Server, thus providing one of the largest collections of aeronautical and space science STI in the world. Results are published in both non-NASA channels and by NASA in the NASA STI Report Series, which includes the following report types:

- TECHNICAL PUBLICATION. Reports of completed research or a major significant phase of research that present the results of NASA programs and include extensive data or theoretical analysis. Includes compilations of significant scientific and technical data and information deemed to be of continuing reference value. NASA counterpart of peer-reviewed formal professional papers but has less stringent limitations on manuscript length and extent of graphic presentations.

- TECHNICAL MEMORANDUM. Scientific and technical findings that are preliminary or of specialized interest, e.g., quick release reports, working papers, and bibliographies that contain minimal annotation. Does not contain extensive analysis.

- CONTRACTOR REPORT. Scientific and technical findings by NASA-sponsored contractors and grantees.
- CONFERENCE PUBLICATION. Collected papers from scientific and technical conferences, symposia, seminars, or other meetings sponsored or cosponsored by NASA.

- SPECIAL PUBLICATION. Scientific, technical, or historical information from NASA programs, projects, and missions, often concerned with subjects having substantial public interest.

- TECHNICAL TRANSLATION. Englishlanguage translations of foreign scientific and technical material pertinent to NASA's mission.

Specialized services also include creating custom thesauri, building customized databases, organizing and publishing research results.

For more information about the NASA STI program, see the following:

- Access the NASA STI program home page at http://www.sti.nasa.gov

- E-mail your question via the Internet to help@ sti.nasa.gov

- Fax your question to the NASA STI Help Desk at 443-757-5803

- Telephone the NASA STI Help Desk at 443-757-5802

- Write to: NASA Center for AeroSpace Information (CASI) 7115 Standard Drive Hanover, MD 21076-1320 
NASA/TM-2010-216076

Optimal Tuner Selection for Kalman Filter-Based Aircraft Engine Performance Estimation

Donald L. Simon and Sanjay Garg

Glenn Research Center, Cleveland, Ohio

Prepared for

Turbo Expo 2009

sponsored by the American Society of Mechanical Engineers

Orlando, Florida, June 8-12, 2009

National Aeronautics and

Space Administration

Glenn Research Center

Cleveland, Ohio 44135 


\section{Acknowledgments}

This research was conducted under the NASA Aviation Safety Program, Integrated Vehicle Health

Management Project. The authors graciously acknowledge Jonathan Litt for his support and insightful discussions which led to the development of this work.

Trade names and trademarks are used in this report for identification only. Their usage does not constitute an official endorsement, either expressed or implied, by the National Aeronautics and Space Administration.

Level of Review: This material has been technically reviewed by technical management.

Available from

NASA Center for Aerospace Information 7115 Standard Drive

Hanover, MD 21076-1320
National Technical Information Service 5285 Port Royal Road Springfield, VA 22161

Available electronically at http://gltrs.grc.nasa.gov 


\title{
Optimal Tuner Selection for Kalman Filter-Based Aircraft Engine Performance Estimation
}

\author{
Donald L. Simon and Sanjay Garg \\ National Aeronautics and Space Administration \\ Glenn Research Center \\ Cleveland, Ohio 44135
}

\begin{abstract}
A linear point design methodology for minimizing the error in on-line Kalman filter-based aircraft engine performance estimation applications is presented. This technique specifically addresses the underdetermined estimation problem, where there are more unknown parameters than available sensor measurements. A systematic approach is applied to produce a model tuning parameter vector of appropriate dimension to enable estimation by a Kalman filter, while minimizing the estimation error in the parameters of interest. Tuning parameter selection is performed using a multi-variable iterative search routine which seeks to minimize the theoretical mean-squared estimation error. This paper derives theoretical Kalman filter estimation error bias and variance values at steady-state operating conditions, and presents the tuner selection routine applied to minimize these values. Results from the application of the technique to an aircraft engine simulation are presented and compared to the conventional approach of tuner selection. Experimental simulation results are found to be in agreement with theoretical predictions. The new methodology is shown to yield a significant improvement in on-line engine performance estimation accuracy.
\end{abstract}

\section{Introduction}

An emerging approach in the field of aircraft engine controls and health management is the inclusion of real-time on-board models for the in-flight estimation of engine performance variations (Refs. 1, 2, and 3). This technology, typically based on Kalman filter concepts, enables the estimation of unmeasured engine performance parameters which can be directly utilized by controls, prognostics and health management applications. A challenge which complicates this practice is the fact that an aircraft engine's performance is affected by its level of degradation, generally described in terms of unmeasurable health parameters such as efficiencies and flow capacities related to each major engine module. Through Kalman filter-based estimation techniques, the level of engine performance degradation can be estimated, given that there are at least as many sensors as parameters to be estimated. However, in an aircraft engine the number of sensors available is typically less than the number of health parameters presenting an under-determined estimation problem. A common approach to address this shortcoming is to estimate a sub-set of the health parameters, referred to as model tuning parameters. While this approach enables on-line Kalman filter-based estimation, it can result in "smearing" the effects of un-estimated health parameters onto those which are estimated, and in turn introduce error in the accuracy of overall model-based performance estimation applications. Recently, Litt (Ref. 4) presented an approach based on singular value decomposition which selects a model tuning parameter vector of low-enough dimension to be estimated by a Kalman filter. The model tuning parameter vector, $q$, was constructed as a linear combination of all health parameters, $h$, given by

$$
q=V^{*} h
$$

where the transformation matrix, $V^{*}$, is selected applying singular value decomposition to capture the overall effect of the larger set of health parameters on the engine variables as closely as possible in the least squares sense. In this paper a new linear point design technique which applies a systematic approach to optimal tuning parameter selection will be presented. This technique, like the one presented in Reference 4, also defines a transformation matrix, $V^{*}$, used to construct a tuning parameter vector which is a linear combination of all health parameters, and of low enough dimension to enable Kalman filter estimation. The new approach optimally selects the transformation matrix, $V^{*}$, to minimize the theoretical steady-state estimation error in the engine performance parameters of interest. There is no known closed form solution for optimally selecting $V^{*}$ to satisfy this objective. Therefore, a multivariable iterative search routine is applied to perform this function.

The remaining sections of this paper are organized as follows. First, the mathematical formulation of the parameter estimation problem is presented, and theoretical estimation error values are derived assuming linear, steady-state operating conditions. The theoretical estimation error information is directly used by the iterative search routine applied to optimally select the Kalman filter tuning parameter vector, which is described next. Example estimation results from the application of the new methodology to an aircraft turbofan engine simulation are then presented and compared to the conventional approach of tuning parameter selection, the SVD tuner selection approach presented in Reference 4, and the maximum a posteriori performance estimation approach commonly applied for off-line (ground-based) aircraft engine gas path analysis applications (Refs. 5 and 6). 
After the example, there is a discussion of practical considerations for applying the method, and a discussion of future work. Finally, conclusions are presented.

\section{Nomenclature}

$$
\begin{aligned}
& A, A_{x h}, A_{x q} \\
& B, B_{x h}, B_{x q}, \\
& C, C_{x h}, C_{x q}, \\
& D, F, F_{x h}, F_{x q}, G, \\
& L, M, N \\
& \text { C-MAPSS }
\end{aligned}
$$

Fn$$
G_{x h}, G_{x}, G_{h}, G_{z}
$$$$
H
$$

$\mathrm{HPC}$

HPT$$
\text { I }
$$$$
K_{\infty}
$$$$
\text { LPC }
$$$$
\text { LPT }
$$$$
\text { MAP }
$$$$
\mathrm{Nf}
$$$$
\mathrm{Nc}
$$$$
P_{h}, P_{z}
$$$$
\text { P24 }
$$$$
\text { Ps30 }
$$$$
P_{\hat{x} \hat{h}, k}, P_{\hat{x} \hat{q}, k}, P_{\hat{z}, k}
$$$$
P_{\infty}
$$$$
Q, Q_{x h}, Q_{x q}
$$$$
R
$$$$
\text { SmLPC }
$$$$
\text { T24 }
$$$$
\text { T30 }
$$$$
\text { T40 }
$$$$
\mathrm{T} 48
$$$$
\text { T50 }
$$$$
V^{*}
$$$$
\text { VSV }
$$$$
\text { VBV }
$$$$
\text { Wf }
$$$$
W_{z}
$$$$
h_{k}
$$

$q_{k}$

$u_{k}$

$v_{k}$

$w_{k}, w_{h, k}, w_{x h, k}$

$x_{k}$

$x_{x h, k}$

$x_{x q, k}$

$y_{k}$

$z_{k}$

$\varepsilon_{x q, k}$

Commercial Modular Aero-Propulsion

System Simulation

net thrust

estimation bias matrices

matrix which relates health parameter effects to steady-state engine outputs

high pressure compressor

high pressure turbine

identity matrix

Kalman filter gain

low pressure compressor

low pressure turbine

maximum a posteriori

fan speed

core speed

health \& auxiliary parameter covariance matrices

HPC inlet total pressure

HPC exit static pressure

covariance matrices of estimated

parameters

Kalman filter state estimation covariance matrix

process noise covariance matrices

measurement noise covariance matrix

LPC stall margin

HPC inlet total temperature

HPC exit total temperature

combustor exit temperature

exhaust gas temperature

LPT exit temperature

transformation matrix relating $h_{k}$ to $q_{k}$

variable stator vane

variable bleed valve

fuel flow

auxiliary parameter weighting matrix

health parameter vector

\section{Subscripts}

Kalman filter tuning parameter vector actuator command vector measurement noise vector process noise vectors state vector augmented state vector $\left(x_{k}\right.$ and $\left.h_{k}\right)$ reduced order state vector ( $x_{k}$ and $q_{k}$ ) vector of measured outputs vector of unmeasured (auxiliary) outputs residual vector (estimate minus its expected value)

\section{$k$}

$x h$

$x q$

sS

\section{Superscripts}

$\wedge$

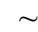

$-$

$\mathrm{T}$

\section{Operators}

$\begin{array}{ll}E[\cdot] & \text { expected value of argument } \\ \operatorname{tr}\{\cdot\} & \text { trace of matrix } \\ \operatorname{SSEE}(\cdot) & \text { sum of squared estimation errors } \\ \operatorname{WSSEE}(\cdot) & \text { weighted sum of squared estimation } \\ \|\cdot\|_{F} & \text { errors } \\ & \text { matrix Frobenius norm }\end{array}$

\section{Problem Formulation}

The discrete linear time-invariant engine state space equations about a linear design point are given as

$$
\begin{aligned}
& x_{k+1}=A x_{k}+B u_{k}+L h_{k}+w_{k} \\
& y_{k}=C x_{k}+D u_{k}+M h_{k}+v_{k} \\
& z_{k}=F x_{k}+G u_{k}+N h_{k}
\end{aligned}
$$

where $k$ is the time index, $x$ is the vector of state variables, $u$ is the vector of control inputs, $y$ is the vector of measured outputs, and $z$ is the vector of auxiliary (unmeasured) model outputs. The vector $h$ represents the engine health parameters, 
which induce shifts in other variables as the health parameters deviate from their nominal values. The vectors $w$ and $v$ are uncorrelated zero-mean white noise input sequences. $Q$ will be used to denote the covariance of $w$, and $R$ to denote the covariance of $v$. The matrices $A, B, C, D, F, G, L, M$, and $N$ are of appropriate dimension. The health parameters, represented by the vector $h$, are unknown inputs to the system. They may be treated as a set of biases, and are thus modeled without dynamics. With this interpretation Eq. (2) can be written as:

$$
\begin{aligned}
& {\left[\begin{array}{l}
x_{k+1} \\
h_{k+1}
\end{array}\right]=\underbrace{\left[\begin{array}{lr}
A & L \\
0 & I
\end{array}\right]}_{A_{x h}} \underbrace{\left[\begin{array}{c}
x_{k} \\
h_{k}
\end{array}\right]}_{x_{x h, k}}+\underbrace{\left[\begin{array}{c}
B \\
0
\end{array}\right]}_{B_{x h}} u_{k}+\underbrace{\left[\begin{array}{c}
w_{k} \\
w_{h, k}
\end{array}\right]}_{w_{x h, k}}} \\
& =A_{x h} x_{x h, k}+B_{x h} u_{k}+w_{x h, k} \\
& y_{k}=\underbrace{\left[\begin{array}{ll}
C & M
\end{array}\right]}_{C_{x h}} \underbrace{\left[\begin{array}{c}
x_{k} \\
h_{k}
\end{array}\right]}_{x_{x h, k}}+D u_{k}+v_{k} \\
& =C_{x h} x_{x h, k}+D u_{k}+v_{k} \\
& z_{k}=\underbrace{\left[\begin{array}{ll}
F & N
\end{array}\right]}_{F_{x h}} \underbrace{\left[\begin{array}{c}
x_{k} \\
h_{k}
\end{array}\right]}_{x_{x h, k}}+G u_{k} \\
& =F_{x h} x_{x h, k}+G u_{k}
\end{aligned}
$$

The vector $w_{x h}$ is zero-mean white noise associated with the augmented state vector, $\left[x^{\mathrm{T}} h^{\mathrm{T}}\right]^{\mathrm{T}}$, with a covariance of $Q_{x h} \cdot w_{x h}$ consists of the original state process noise, $w$, concatenated with the process noise associated with the health parameter vector, $w_{h}$.

$$
w_{x h, k}=\left[\begin{array}{c}
w_{k} \\
w_{h, k}
\end{array}\right]
$$

The eigenvalues of $A_{x h}$ consist of the original eigenvalues of $A$ plus an additional $\operatorname{dim}(h)$ eigenvalues located at 1.0 on the unit circle due to the augmentation. Thus, the new augmented system given in Eq. (3) has at least as many eigenvalues located on the unit circle as there are elements of $h$. Once the $h$ vector is appended to the state vector, it may be directly estimated, provided that the realization in Eq. (3) is observable. Using this formulation, the number of health parameters that can be estimated is limited to the number of sensors, the dimension of $y$ (Ref. 7). Since in an aircraft gas turbine engine there are usually fewer sensors than health parameters, the problem becomes one of choosing the best set of tuners for the application. This paper presents a systematic methodology for the optimal selection of a model tuning parameter vector, $q$, of low-enough dimension to be estimated by a Kalman filter, while minimizing the estimation error in the model variables of interest. The following sub-sections will cover the steps in the problem setup. This includes construction of the reduced-order state space model, formulation of the Kalman filter estimator, calculation of the mean sum of squared estimation errors, and optimal selection of the transformation matrix to minimize the estimation error.

\section{Reduced-Order State Space Model}

The model tuning parameter vector, $q$, is constructed as a linear combination of all health parameters, $h$, given by

$$
q=V^{*} h
$$

where $q \in \mathbb{R}^{m}, h \in \mathbb{R}^{p}, m<p$, and $V^{*}$ is an $m \times p$ transformation matrix of rank $m$, applied to construct the tuning parameter vector. An approximation of the health parameter vector, $\hat{h}$, can be obtained as

$$
\hat{h}=V^{* \dagger} q
$$

where $V^{* \dagger}$ is the pseudo-inverse of $V^{*}$. Substituting Eq. (6) into Eq. (3) yields the following reduced order state space equations which will be used to formulate the Kalman filter

$$
\begin{aligned}
& {\left[\begin{array}{c}
x_{k+1} \\
q_{k+1}
\end{array}\right]=\underbrace{\left[\begin{array}{cc}
A & L V^{*} \dagger \\
0 & I
\end{array}\right]}_{A_{x q}} \underbrace{\left[\begin{array}{c}
x_{k} \\
q_{k}
\end{array}\right]}_{x_{x q, k}}+\underbrace{\left[\begin{array}{c}
B \\
0
\end{array}\right]}_{B_{x q}} u_{k}+\underbrace{\left[\begin{array}{c}
w_{k} \\
w_{q, k}
\end{array}\right]}_{w_{x q, k}}} \\
& =A_{x q} x_{x q, k}+B_{x q} u_{k}+w_{x q, k} \\
& y_{k}=\underbrace{\left[\begin{array}{ll}
C & M V^{* \dagger}
\end{array}\right]}_{C_{x q}} \underbrace{\left[\begin{array}{c}
x_{k} \\
q_{k}
\end{array}\right]}_{x_{x q, k}}+D u_{k}+v_{k} \\
& =C_{x q} x_{x q, k}+D u_{k}+v_{k} \\
& z_{k}=\underbrace{\left[\begin{array}{cc}
F & N V^{* \dagger}
\end{array}\right]}_{F_{x q}} \underbrace{\left[\begin{array}{c}
x_{k} \\
q_{k}
\end{array}\right]}_{x_{x q, k}}+G u_{k} \\
& =F_{x q} x_{x q, k}+G u_{k}
\end{aligned}
$$

The state process noise, $w_{x q}$, and its associated covariance, $Q_{x q}$, for the reduced order system are calculated as

$$
\begin{aligned}
& w_{x q, k}=\left[\begin{array}{cc}
I & 0 \\
0 & V^{*}
\end{array}\right] w_{x h, k}=\left[\begin{array}{cc}
I & 0 \\
0 & V^{*}
\end{array}\right]\left[\begin{array}{c}
w_{k} \\
w_{h, k}
\end{array}\right] \\
& Q_{x q}=\left[\begin{array}{cc}
I & 0 \\
0 & V^{*}
\end{array}\right] Q_{x h}\left[\begin{array}{cc}
I & 0 \\
0 & V^{*}
\end{array}\right]^{T}
\end{aligned}
$$

\section{Kalman Filter Formulation}

In this study, steady-state Kalman filtering is applied. This means that while the Kalman filter is a dynamic system, the state estimation error covariance matrix and the Kalman gain matrix are invariant-instead of updating these matrices each 
time step they are held constant. Given the reduced order linear state space equations shown in Eq. (7), the state estimation error covariance matrix, $P_{\infty}$, is calculated by solving the following Ricatti equation (Ref. 8):

$$
\begin{aligned}
P_{\infty}= & A_{x q} P_{\infty} A_{x q}^{T}-A_{x q} P_{\infty} C_{x q}^{T}\left(C_{x q} P_{\infty} C_{x q}^{T}+R\right)^{-1} \ldots \\
& \times C_{x q} P_{\infty} A_{x q}^{T}+Q_{x q}
\end{aligned}
$$

The steady-state Kalman filter gain, $K_{\infty}$, can then be calculated as follows (Ref. 8)

$$
K_{\infty}=P_{\infty} C_{x q}^{T}\left(C_{x q} P_{\infty} C_{x q}^{T}+R\right)^{-1}
$$

and, assuming steady-state, open-loop operation $(u=0)$, the Kalman filter estimator takes the following form

$$
\hat{x}_{x q, k}=A_{x q} \hat{x}_{x q, k-1}+K_{\infty}\left(y_{k}-C_{x q} A_{x q} \hat{x}_{x q, k-1}\right)
$$

The reduced order state vector estimate, $\hat{x}_{x q}$, produced by Eq. (11) can be used to produce an estimate of the augmented state vector, and the auxiliary parameter vector as follows

$$
\begin{aligned}
& \hat{x}_{x h, k}=\left[\begin{array}{cc}
I & 0 \\
0 & V^{* \dagger}
\end{array}\right] \hat{x}_{x q, k} \\
& \hat{z}_{k}=\left[\begin{array}{ll}
F & N V^{* \dagger}
\end{array}\right] \hat{x}_{x q, k}
\end{aligned}
$$

\section{Analytical Derivation of Estimation Error}

The estimation errors in $\hat{x}_{x h, k}$ and $\hat{z}_{k}$ are defined as the difference between the estimated and actual values $\backslash$

$$
\begin{aligned}
& \tilde{x}_{x h, k}=\hat{x}_{x h, k}-x_{x h, k} \\
& \tilde{z}_{k}=\hat{z}_{k}-z_{k}
\end{aligned}
$$

Due to the under-determined nature of the estimation problem, it will be impossible for the Kalman filter estimator to completely restore all information when transforming $\hat{q}$ into

$\hat{h}$. As such, the Kalman filter will be a biased estimator (i.e. the expected values of $\tilde{x}_{x h, k}$ and $\widetilde{z}_{k}$ will be non-zero). The estimation errors can be considered to consist of two components: An estimation error bias, and an estimation variance. The estimation error bias vectors are equivalent to the mean estimation error vectors defined as

$$
\begin{aligned}
& \overline{\tilde{x}}_{x h, k}=E\left[\tilde{x}_{x h, k}\right]=E\left[\hat{x}_{x h, k}-x_{x h, k}\right] \\
& \overline{\tilde{z}}_{k}=E\left[\tilde{z}_{k}\right]=E\left[\hat{z}_{k}-z_{k}\right]
\end{aligned}
$$

where the operator $E[\bullet]$ represents the expected value of the argument. The variance of the estimates can be found by constructing their respective estimation covariance matrices

$$
\begin{aligned}
& P_{\hat{x} \hat{h}, k}=E\left[\left(\hat{x}_{x h, k}-E\left[\hat{x}_{x h, k}\right]\right)\left(\hat{x}_{x h, k}-E\left[\hat{x}_{x h, k}\right]\right)^{T}\right] \\
& P_{\hat{z}, k}=E\left[\left(\hat{z}_{k}-E\left[\hat{z}_{k}\right]\right)\left(\hat{z}_{k}-E\left[\hat{z}_{k}\right]\right)^{T}\right]
\end{aligned}
$$

Diagonal elements of the covariance matrices will reflect the variance in individual parameter estimates, while off-diagonal elements reflect the covariance between parameter estimates. The overall sum of squared estimation errors (SSEE) can be obtained by combining the estimation error bias and estimation variance information as

$$
\begin{aligned}
& \operatorname{SSEE}\left(\hat{x}_{x h, k}\right)=\overline{\tilde{x}}_{x h, k}^{T} \overline{\tilde{x}}_{x h, k}+\operatorname{tr}\left\{P_{\hat{x} \hat{h}, k}\right\} \\
& \operatorname{SSEE}\left(\hat{z}_{k}\right)=\overline{\tilde{z}}_{k}^{T} \overline{\tilde{z}}_{k}+\operatorname{tr}\left\{P_{\hat{z}, k}\right\}
\end{aligned}
$$

where $\operatorname{tr}\{\bullet\}$ represents the trace (sum of the diagonal elements) of the matrix. In this paper, theoretical values for each error component will be derived assuming steady-state, open-loop $(u=0)$ operating conditions. First, the estimation error bias is derived, followed by a derivation of the estimation variance.

\section{Estimation Error Bias}

The estimation error biases, $\overline{\widetilde{x}}_{x h, k}$ and $\overline{\widetilde{z}}_{k}$, can be analytically derived for an arbitrary health parameter vector, $h$, at steady-state operating conditions. This is done taking advantage of the following expected value properties at steady-state open-loop operating conditions

$$
\begin{aligned}
& E\left[x_{k+1}\right]=E\left[x_{k}\right]=x_{s s} \\
& E\left[h_{k}\right]=h \\
& E\left[x_{x h, k}\right]=x_{x h, s s} \\
& E\left[y_{k}\right]=y_{s s} \\
& E\left[z_{k}\right]=z_{s s} \\
& E\left[u_{k}\right]=0 \\
& E\left[w_{k}\right]=0 \\
& E\left[v_{k}\right]=0 \\
& E\left[\hat{x}_{x q, k}\right]=E\left[\hat{x}_{x q, k-1}\right]=\overline{\hat{x}}_{x q, s s} \\
& E\left[\hat{x}_{x h, k}\right]=\overline{\hat{x}}_{x h, s s} \\
& E\left[\hat{z}_{k}\right]=\overline{\hat{z}}_{s s}
\end{aligned}
$$


where the subscript "ss" denotes steady-state operation. By taking expected values of Eq. (2), $x_{s s}, y_{s s}$ and $z_{s s}$ can be written as functions of the health parameter vector $h$

$$
\begin{aligned}
& E\left[x_{k+1}\right]=A \cdot E\left[x_{k}\right]+B \cdot E\left[u_{k}\right]+L \cdot E\left[h_{k}\right]+E\left[w_{k}\right] \\
& x_{s s}=A x_{s s}+L h \\
& x_{S S}=(I-A)^{-1} L h \\
& E\left[y_{k}\right]=C \cdot E\left[x_{k}\right]+D \cdot E\left[u_{k}\right]+M \cdot E\left[h_{k}\right]+E\left[v_{k}\right] \\
& y_{S S}=C x_{S S}+M h \\
& y_{s s}=\left(C(I-A)^{-1} L+M\right) h \\
& E\left[z_{k}\right]=F \cdot E\left[x_{k}\right]+G \cdot E\left[u_{k}\right]+N \cdot E\left[h_{k}\right] \\
& z_{s s}=F x_{s s}+N h \\
& z_{s s}=\left(F(I-A)^{-1} L+N\right) h
\end{aligned}
$$

Next, by taking expected values of both sides of Eq. (11), the expected value of $\hat{x}_{x q, k}$ can be obtained as a function of $y_{s s}$ :

$$
\begin{aligned}
& E\left[\hat{x}_{x q, k}\right]=A_{x q} \cdot E\left[\hat{x}_{x q, k-1}\right] \ldots \\
& +K_{\infty}\left(E\left[y_{k}\right]-C_{x q} A_{x q} \cdot E\left[\hat{x}_{x q, k-1}\right]\right) \\
& \overline{\hat{x}}_{x q, s s}=A_{x q} \overline{\hat{x}}_{x q, s s}+K_{\infty}\left(y_{s s}-C_{x q} A_{x q} \overline{\hat{x}}_{x q, s s}\right) \\
& \overline{\hat{x}}_{x q, s s}=\left(I-A_{x q}+K_{\infty} C_{x q} A_{x q}\right)^{-1} K_{\infty} y_{s s}
\end{aligned}
$$

Then, making the substitution $y_{s s}=\left(C(I-A)^{-1} L+M\right) h$ given in Eq. (19), the expected steady-state value of $\hat{x}_{x q, k}$ can be written as a function of $h$

$$
\begin{aligned}
\overline{\hat{x}}_{x q, s s}= & \left(I-A_{x q}+K_{\infty} C_{x q} A_{x q}\right)^{-1} \ldots \\
& \times K_{\infty}\left(C(I-A)^{-1} L+M\right) h
\end{aligned}
$$

The steady-state augmented state estimation error bias can then be found, and partitioned into error bias information for the original state vector, $\overline{\widetilde{x}}_{S S}$, and the health parameter vector, $\overline{\widetilde{h}}_{s s}$, by combining Eqs. (12), (14), (18) and (22) to yield

$$
\begin{aligned}
& \overline{\tilde{x}}_{x h, s s}=E\left[\hat{x}_{x h, k}-x_{x h, k}\right] \\
& \overline{\tilde{x}}_{x h, s s}=\overline{\hat{x}}_{x h, s s}-x_{x h, s s} \\
& \overline{\tilde{x}}_{x h, s s}=\left[\begin{array}{l}
\overline{\tilde{x}}_{s s} \\
\tilde{\tilde{h}}_{s s}
\end{array}\right] \\
& =\left[\begin{array}{cc}
I & 0 \\
0 & V^{* \dagger}
\end{array}\right] \overline{\hat{x}}_{x q, s s}-x_{x h, s s} \\
& \overline{\tilde{x}}_{x h, s s}=\left[\begin{array}{l}
\overline{\tilde{x}}_{s s} \\
\tilde{\tilde{h}}_{s s}
\end{array}\right] \\
& =\underbrace{\left[\begin{array}{cc}
{\left[\begin{array}{cc}
I & 0 \\
0 & V^{* \dagger}
\end{array}\right]\left(I-A_{x q}+K_{\infty} C_{x q} A_{x q}\right)^{-1} \ldots} \\
\times K_{\infty}\left[\begin{array}{c}
\left.C(I-A)^{-1} L+M\right] \ldots \\
\end{array}\right]
\end{array}\right.}_{G_{x h}} \underbrace{-\left[\begin{array}{c}
(I-A)^{-1} L \\
I
\end{array}\right]} \\
& \overline{\tilde{x}}_{x h, s s}=\left[\begin{array}{l}
\overline{\tilde{x}}_{s s} \\
\tilde{\tilde{h}}_{s s}
\end{array}\right]=G_{x h} h
\end{aligned}
$$

The steady-state auxiliary parameter estimation error bias can also be derived by combining Eqs. (12), (14), (20), and (22) to yield

$$
\begin{aligned}
& \overline{\tilde{z}}_{s s}=E\left[\hat{z}_{k}-z_{k}\right] \\
& \overline{\tilde{z}}_{s s}=\overline{\hat{z}}_{s s}-z_{s s} \\
& \overline{\tilde{z}}_{S S}=\left[\begin{array}{ll}
F & N V^{* \dagger}
\end{array}\right] \overline{\hat{x}}_{x q, s S}-z_{S S}
\end{aligned}
$$

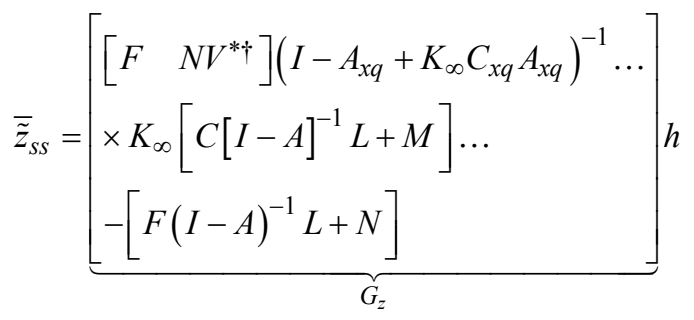

$$
\begin{aligned}
& \overline{\tilde{z}}_{s S}=G_{z} h
\end{aligned}
$$

The estimation error bias equations given in Eqs. (23) and (24) are functions of an arbitrary health parameter vector, $h$. As such they are representative of the parameter estimation error biases in a single engine, at a given point in its lifetime of use where its deterioration is represented by the health parameter vector $h$. The average sum of squared estimation error biases across a fleet of engines can be calculated as 


$$
\begin{aligned}
& \overline{\tilde{x}}_{x h, \text { fleet }}^{2} \equiv E\left[\overline{\tilde{x}}_{x h, s s}^{T} \overline{\tilde{x}}_{x h, s s}\right]=E\left[\operatorname{tr}\left\{\overline{\tilde{x}}_{x h, s s} \overline{\tilde{x}}_{x h, s s}^{T}\right\}\right] \\
& =E\left[\operatorname{tr}\left\{G_{x h} h h^{T} G_{x h}^{T}\right\}\right] \\
& =\operatorname{tr}\{G_{x h} \cdot E \underbrace{\left[h h^{T}\right]}_{P_{h}} G_{x h}^{T}\} \\
& =\operatorname{tr}\left\{G_{x h} P_{h} G_{x h}^{T}\right\} \\
& \overline{\tilde{z}}_{\text {fleet }}^{2} \equiv E\left[\overline{\tilde{z}}_{s S}^{T} \overline{\tilde{z}}_{s S}\right]=E\left[\operatorname{tr}\left\{\overline{\tilde{z}}_{s s} \overline{\tilde{z}}_{s s}^{T}\right\}\right] \\
& =E\left[\operatorname{tr}\left\{G_{z} h h^{T} G_{z}^{T}\right\}\right] \\
& =\operatorname{tr}\{G_{z} \cdot E \underbrace{\left[h h^{T}\right]}_{P_{h}} G_{z}^{T}\} \\
& =\operatorname{tr}\left\{G_{z} P_{h} G_{z}^{T}\right\}
\end{aligned}
$$

where the matrix $P_{h}$, defined as $E\left[h h^{T}\right]$, reflects a priori or historical knowledge of the covariance in the health parameters across all engines. If available, it can be used to predict the sum of squared estimation errors biases as shown in (25) and (26).

\section{Estimation Variance}

Next, derivations are presented for the augmented state estimate and auxiliary parameter estimate covariance matrices, $P_{\hat{x} \hat{h}, k}$ and $P_{\hat{z}, k}$, respectively. These matrices will be calculated as a function of the reduced-order state vector estimation covariance matrix, $P_{\hat{x} \hat{q}, k}$, which is defined as

$$
P_{\hat{x} \hat{q}, k}=E[\underbrace{\left(\hat{x}_{x q, k}-E\left[\hat{x}_{x q, k}\right]\right)}_{\varepsilon_{x q, k}} \underbrace{\left(\hat{x}_{x q, k}-E\left[\hat{x}_{x q, k}\right]\right)^{T}}_{\varepsilon_{x q, k}^{T}}]
$$

where the vector $\varepsilon_{x q, k}$ is defined as the residual between $\hat{x}_{x q, k}$ at time $k$ and its expected value. Since $E\left[\hat{x}_{x q, k}\right]=\overline{\hat{x}}_{x q, s s}, \varepsilon_{x q, k}$ can be obtained by subtracting Eq. (21) from Eq. (11)

$$
\begin{aligned}
& \varepsilon_{x q, k}=\hat{x}_{x q, k}-E\left[\hat{x}_{x q, k}\right] \\
& =\hat{x}_{x q, k}-\overline{\hat{x}}_{x q, s s} \\
& =\underbrace{A_{x q} \hat{x}_{x q, k-1}+K_{\infty}\left(y_{k}-C_{x q} A_{x q} \hat{x}_{x q, k-1}\right)}_{\hat{x}_{x q, k}} \cdots \\
& -\underbrace{\left(A_{x q} \overline{\hat{x}}_{x q, s s}+K_{\infty}\left(y_{s s}-C_{x q} A_{x q} \overline{\hat{x}}_{x q, s s}\right)\right)}_{\overline{\hat{x}}_{x q, s s}} \\
& =\left(A_{x q}-K_{\infty} C_{x q} A_{x q}\right)\left(\hat{x}_{x q, k-1}-\overline{\hat{x}}_{x q, s s}\right) \\
& +K_{\infty}\left(y_{k}-y_{s s}\right)
\end{aligned}
$$

Making the substitutions $\varepsilon_{x q, k-1}=\hat{x}_{x q, k-1}-\overline{\hat{x}}_{x q, s s}$, and $v_{k}=y_{k}-y_{s s}$ yields

$$
\varepsilon_{x q, k}=\left(A_{x q}-K_{\infty} C_{x q} A_{x q}\right) \varepsilon_{x q, k-1}+K_{\infty} v_{k}
$$

The estimation covariance matrix $P_{\hat{x} \hat{q}, k}$ is then calculated as

$$
\begin{aligned}
P_{\hat{x} \hat{q}, k}= & E\left[\varepsilon_{x q, k} \varepsilon_{x q, k}^{T}\right] \\
= & {\left[A_{x q}-K_{\infty} C_{x q} A_{x q}\right] E\left[\varepsilon_{x q, k-1} \varepsilon_{x q, k-1}^{T}\right] \ldots } \\
& \times\left[A_{x q}-K_{\infty} C_{x q} A_{x q}\right]^{T} \ldots \\
& +\left[A_{x q}-K_{\infty} C_{x q} A_{x q}\right] E\left[\varepsilon_{x q, k-1} v_{k}^{T}\right] K_{\infty}^{T} \ldots \\
& +K_{\infty} E\left[v_{k} \varepsilon_{x q, k-1}^{T}\right]\left[A_{x q}-K_{\infty} C_{x q} A_{x q}\right]^{T} \ldots \\
& +K_{\infty} E\left[v_{k} v_{k}^{T}\right] K_{\infty}^{T}
\end{aligned}
$$

The substitutions $E\left[\varepsilon_{x q, k-1} \varepsilon_{x q, k-1}^{T}\right]=P_{\hat{x} \hat{x}, k-1}$, and $E\left[v_{k} v_{k}^{T}\right]=R$ can be made in the above equation. Since $\varepsilon_{x q, k-1}$ and $v_{k}^{T}$ are uncorrelated, the substitution $E\left[\varepsilon_{x q, k-1} v_{k}^{T}\right]=E\left[v_{k} \varepsilon_{x q, k-1}^{T}\right]=0$ can also be made, producing

$$
\begin{aligned}
P_{\hat{x} \hat{q}, k}= & {\left[A_{x q}-K_{\infty} C_{x q} A_{x q}\right] P_{\hat{x} \hat{q}, k-1}\left[A_{x q}-K_{\infty} C_{x q} A_{x q}\right]^{T} } \\
& +K_{\infty} R K_{\infty}^{T}
\end{aligned}
$$

At steady-state operating conditions $P_{\hat{x} \hat{q}, k-1}=P_{\hat{x} \hat{q}, k}$. Making this substitution in (31) produces the following Ricatti equation which can be solved for $P_{\hat{x} \hat{q}, k}$ :

$$
\begin{aligned}
P_{\hat{x} \hat{q}, k}= & {\left[A_{x q}-K_{\infty} C_{x q} A_{x q}\right] P_{\hat{x} \hat{q}, k}\left[A_{x q}-K_{\infty} C_{x q} A_{x q}\right]^{T} } \\
& +K_{\infty} R K_{\infty}^{T}
\end{aligned}
$$


It should be noted that $P_{\hat{x} \hat{q}, k}$ obtained by solving (32) will be identical to $P_{\infty}$ produced via Eq. (9) if the system's actual state process noise covariance is identical to the $Q_{x q}$ assumed in the design of the Kalman filter. However, $Q$ is often treated as a Kalman filter design parameter to provide acceptable dynamic response. For the purpose of this derivation, we have assumed a steady-state operating condition where the state variables and health parameters are invariant, and thus the actual system process noise is zero (i.e., $w_{x h, k}=0$ ). In this case $P_{\hat{x} \hat{q}, k}$ will not equal $P_{\infty}$. Once $P_{\hat{x} \hat{q}, k}$ is obtained, it can be used to calculate $P_{\hat{x} \hat{h}, k}$, the covariance of $\hat{x}_{x h, k}$, which is defined as $E\left[\left(\hat{x}_{x h, k}-E\left[\hat{x}_{x h, k}\right]\right)\left(\hat{x}_{x h, k}-E\left[\hat{x}_{x h, k}\right]\right)^{T}\right]$

$$
P_{\hat{x} \hat{h}, k}=\left[\begin{array}{cc}
I & 0 \\
0 & V^{* \dagger}
\end{array}\right] P_{\hat{x} \hat{q}, k}\left[\begin{array}{cc}
I & 0 \\
0 & V^{* \dagger}
\end{array}\right]^{T}
$$

The augmented state vector estimation covariance given in Eq. (33) can be partitioned into covariance information for the original state vector, $P_{\hat{x}, k}$ (upper left corner of the $P_{\hat{x} \hat{h}, k}$ matrix), and the health parameter vector, $P_{\hat{h}, k}$ (lower right corner of the $P_{\hat{x} \hat{h}, k}$ matrix)

$$
P_{\hat{x} \hat{h}, k}=\left[\begin{array}{cc}
P_{\hat{x}, k} & \cdots \\
\cdots & P_{\hat{h}, k}
\end{array}\right]
$$

The $P_{\hat{x} \hat{q}, k}$ matrix from Eq. (32) can also be used to calculate $P_{\hat{z}, k}$, the covariance in the estimation of $z_{k}$, which is equivalent to $E\left[\left(\hat{z}_{k}-E\left[\hat{z}_{k}\right]\right)\left(\hat{z}_{k}-E\left[\hat{z}_{k}\right]\right)^{T}\right]$

$$
P_{\hat{z}, k}=\left[\begin{array}{ll}
F & N V^{* \dagger}
\end{array}\right] P_{\hat{x} \hat{q}, k}\left[\begin{array}{ll}
F & N V^{* \dagger}
\end{array}\right]^{T}
$$

The variance in the estimates $\hat{x}_{x h, k}$ and $\hat{z}_{k}$ can be obtained from the diagonals of the covariance matrices produced by (33) and (35) respectively.

\section{Sum of Squared Estimation Errors}

Once Eqs. (25), (26), (33) and (35) are obtained, they may be used to analytically calculate the mean sum of squared estimation errors over all engines by combining the respective estimation error bias and estimation variance information as previously shown in Eq. (16). The mean augmented state vector sum of squared estimation errors, $\operatorname{SSEE}\left(\hat{x}_{x h \text {,fleet }}\right)$, and the mean auxiliary parameter vector sum of squared estimation errors, $\operatorname{SSEE}\left(\hat{z}_{\text {fleet }}\right)$, become

$$
\begin{aligned}
& \operatorname{SSEE}\left(\hat{x}_{x h, \text { fleet }}\right)=\overline{\tilde{x}}_{x h, \text { fleet }}^{2}+\operatorname{tr}\left\{P_{\hat{x} \hat{h}, k}\right\} \\
& =\operatorname{tr}\left\{G_{x h} P_{h} G_{x h}^{T}+P_{\hat{x} \hat{h}, k}\right\} \\
& \operatorname{SSEE}\left(\hat{z}_{\text {fleet }}\right)=\overline{\tilde{z}}_{\text {fleet }}^{2}+\operatorname{tr}\left\{P_{\hat{z}, k}\right\} \\
& =\operatorname{tr}\left\{G_{z} P_{h} G_{z}^{T}+P_{\hat{z}, k}\right\}
\end{aligned}
$$

If required, a weighted sum approach can be applied to normalize the contributions of individual auxiliary parameter estimation errors. This is often necessary as there may be several orders of magnitude difference between the auxiliary parameters of interest. A weighted sum approach prevents domination by individual parameters. In this study a diagonal auxiliary parameter weighting matrix, $W_{z}$, is applied based on the inverse of auxiliary parameter variance (obtained from the main diagonal of the auxiliary parameter covariance matrix, $\left.P_{z}\right)$

$$
\begin{aligned}
P_{z} & =\left[F(I-A)^{-1} L+N\right] P_{h}\left[F(I-A)^{-1} L+N\right]^{T} \\
W_{z} & =\left[\begin{array}{ccc}
P_{z, 11} & 0 & 0 \\
0 & \ddots & 0 \\
0 & 0 & P_{z, i i}
\end{array}\right]^{-1}
\end{aligned}
$$

$W_{z}$ is then applied to calculate of a "weighted" sum of auxiliary parameter squared estimation errors given as

$$
\operatorname{WSSEE}\left(\hat{z}_{\text {fleet }}\right)=\operatorname{tr}\left\{W_{z}\left[G_{z} P_{h} G_{z}^{T}+P_{\hat{z}, k}\right]\right\}
$$

From Eqs. (23), (24), (33) and (35) it can be observed that both bias and variance are affected by the selection of the transformation matrix, $V^{*}$. The sum of squared estimation error terms derived in this section give rise to an optimization problem: selecting $V^{*}$ to minimize the squared estimation error in the Kalman filter produced parameter estimates. This could include health parameter estimates, auxiliary parameter estimates, or a combination of parameters. Although there is no known closed form solution for optimally selecting the $V^{*}$ matrix to satisfy the objective of minimizing estimation errors, a multi-parameter iterative search method has been developed to perform this task, and will be described in the next section.

\section{Optimal Transformation Matrix Selection}

Prior to initiating the search for an optimal $V^{*}$, specific system design information must be defined or obtained. This includes:

- $\quad$ Specifying the auxiliary parameters to be estimated

- Generating system state space equations at a fleet average (50 percent deteriorated) engine trim point 
- Defining measurement noise covariance matrix, $R$

- Defining augmented state process noise covariance matrix, $Q_{x h}$

- Defining fleet average health parameter covariance, $P_{h}$

Some additional clarification is provided regarding the selection of $P_{h}$ and $Q_{x h}$ as the distinction between these two covariance matrices may not be immediately obvious. $P_{h}$ defines the expected health parameter covariance across all engines. It may be based on past knowledge gained from engine gas path analysis programs and/or historical studies of engine module performance deterioration. Conversely, $Q_{x h}$ defines the expected process noise covariance in the state variables and health parameters of an individual engine, at a single discrete time step, $k$. The selection of $Q_{x h}$ will directly impact the dynamic response and the variance of the estimates generated by the Kalman filter, and to a large extent $Q_{x h}$ is treated as a design parameter.

After the necessary system information has been obtained, the search for an optimal transformation matrix to minimize the Kalman filter sum of squared estimation errors can commence. This is performed using the lsqnonlin function of the Matlab (The MathWorks, Inc.) Optimization Toolbox. This function applies an iterative search to find the least squares solution of a user-specified multivariable optimization problem. A flow chart depicting the steps in this optimal iterative search is shown in Figure 1, and a further description of each step is given below.

1. Upon startup, an initial random guess of $V^{*}$ is generated. It is selected such that the matrix Frobenius norm $\left\|V^{*}\right\|_{F}=1$. This requirement is applied to help prevent convergence to a poorly scaled solution.

2. Construct reduced order state-space model, (Eq. (7)).

3. Formulate Kalman filter

a. Calculate estimation covariance matrix, $P_{\infty}$ (Eq. (9))

b. Calculate the Kalman gain matrix, $\mathrm{K}_{\infty}$, (Eq. (10))

4. Calculate sum of squared estimation errors (Eq. (36)), or weighted sum of squared estimation errors (Eq. (38))

5. On each iteration the change in SSEE (or WSSEE) relative to the previous iteration is assessed to determine if convergence within a user specified tolerance has been achieved.

a. If converged, skip step 6 and proceed to step 7

b. If not converged, proceed to step 6 to update $V^{*}$

6. $V^{*}$ is updated via the Matlab lsqnonlin function, again requiring that $\left\|V^{*}\right\|_{F}=1$, and the process returns to step 2

7. Upon convergence, the optimization routine returns the optimal value of $V^{*}$, and ends.

Experience has shown that the transformation matrix returned by the optimization routine is not unique - different matrices can be found which produce a global minimum of the

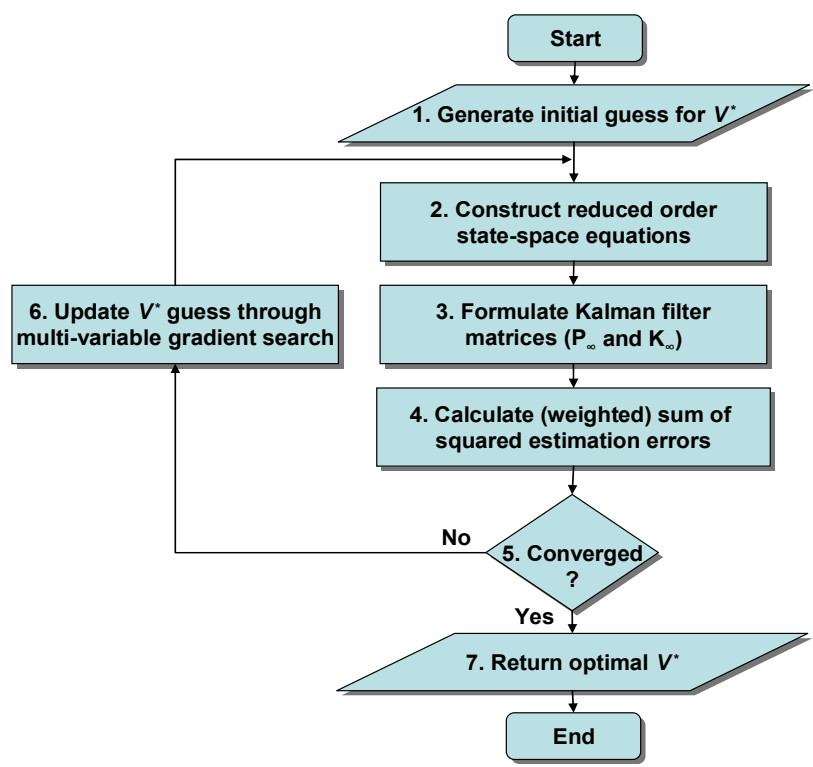

Figure 1.-Flowchart of $V^{\star}$ Iterative Optimal Search

objective function. Experience has also shown that the optimization routine will usually return a $V^{*}$ matrix which satisfies, or nearly satisfies (i.e., within 5 percent), the global minimum of the objective function. However, in order to guard against potential convergence to a local minimum, it is prudent to run the optimization routine multiple times, each time starting with a different initial guess for $V^{*}$. This is only to assure the designer that the global minimum is achieved, not to produce a consistent $V^{*}$. It should be emphasized that the optimal search for $V^{*}$ is only conducted off-line during the estimator design process. This calculation is not conducted as part of the on-line real-time Kalman filter implementation, and thus places no additional computational burden upon it.

\section{Turbofan Engine Example}

A linearized cruise operating point extracted from the NASA Commercial Modular Aero-Propulsion System Simulation (C-MAPSS) high-bypass turbofan engine model (Ref. 9) is used to evaluate the new systematic tuner selection methodology. The linear model has two state variables, ten health parameters, and three control inputs, all shown in Table 1. The model's seven sensed outputs, and corresponding sensor noise standard deviation, are shown in Table 2. The auxiliary output parameters of interest to be estimated are shown in

Table 3. The linear model is used as the truth model for this application example. The model is run open-loop, so all control inputs remain at 0 , i.e., they do not deviate from the trim value for the linear model and no actuator bias is present. Deviations in all ten health parameters are assumed to be uncorrelated, and randomly shifted from their trim conditions with a standard deviation of \pm 0.02 ( \pm 2 percent). Since a 
parameter's variance is equal to its standard deviation squared, the health parameter covariance matrix, $P_{h}$, is defined as a diagonal matrix with all diagonal elements equal to 0.0004 .

Next, the estimation accuracy of the systematic approach for selecting Kalman filter tuning parameters will be compared to the conventional approach of selecting a sub-set of health parameters to serve as tuners (the seven health parameters denoted with "*" in Table 1), and the singular value decomposition approach to tuner selection introduced by Litt in Reference 4. Table 4 shows a comparison of the theoretically predicted estimation errors (squared bias, variance, and total squared error) and experimentally obtained squared estimation errors for each of the three tuner selection approaches. T40 and T50 estimation errors are shown in squared degrees Rankine, and Fn and SmLPC estimation errors are shown in squared percent net thrust and squared percent stall margin respectively. The experimental results were obtained through a Monte Carlo simulation analysis where the health parameters varied over a random distribution in accordance with the covariance matrix, $P_{h}$. The test cases were concatenated to produce a single time history input which was provided to the C-MAPSS linear discrete state space model given in Eq. (2), with an update rate of $15 \mathrm{~ms}$. Each individual health parameter test case lasted $30 \mathrm{~s}$.

TABLE 1.-STATE VARIABLES, HEALTH PARAMETERS, AND ACTUATORS

\begin{tabular}{|c|l|l|}
\hline State variables & Health parameters & \multicolumn{1}{c|}{ Actuators } \\
\hline Nf—-fan speed & Fan efficiency & Wf_fuel flow \\
Nc_core speed & Fan flow capacity* & VSV_variable stator vane \\
& LPC efficiency* & VBV_-variable bleed valve \\
& LPC flow capacity & \\
& HPC efficiency* & \\
& HPC flow capacity* & \\
& HPT efficiency* & \\
& HPT flow capacity* & \\
& LPT efficiency & \\
& LPT flow capacity* & \\
\hline
\end{tabular}

*Health parameters selected as tuners in conventional estimation approach, (identical to sub-set of health parameters selected as tuners in Ref. 4)

TABLE 2.-SENSED OUTPUTS AND STANDARD DEVIATION AS PERCENT OF OPERATING POINT TRIM VALUES

\begin{tabular}{|c|c|}
\hline Sensed output & $\begin{array}{r}\text { Standard deviation } \\
(\%)\end{array}$ \\
\hline $\mathrm{Nf}$-fan speed & ․․․․……………… \\
\hline $\mathrm{Nc}$ - core spee & .0 .25 \\
\hline P24-HPC inle & .....0.50 \\
\hline $\mathrm{T} 24-\mathrm{HPC}$ & $\ldots 0.75$ \\
\hline Ps $30-$ HPC ex & 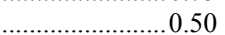 \\
\hline T30- HPC exi & $\ldots 0.75$ \\
\hline T48-Exhaust & $\ldots .0 .75$ \\
\hline
\end{tabular}

TABLE 3.-ESTIMATED AUXILIARY PARAMETERS

Auxiliary parameter

T40 - Combustor exit temperature

T50-LPT exit temperature

Fn-Net thrust

SmLPC-LPC stall margin
At the completion of each $30 \mathrm{~s}$ test case, the health parameter vector input instantaneously transitioned to the next test case. A total of $37530 \mathrm{~s}$ test cases were evaluated, resulting in an 11,250 $\mathrm{s}$ input time history. Three separate Kalman filters were implemented using the three tuner selection approaches. The experimental estimation errors were determined by calculating the mean squared error between estimated and actual values during the last $10 \mathrm{~s}$ of each $30 \mathrm{~s}$ test case. The error calculation is based on only the last $10 \mathrm{~s}$ so that engine model outputs and Kalman estimator outputs have reached a quasi-steady-state operating condition prior to calculating the error. This ensures that the experimental results are consistent with the theoretically predicted estimation errors which were derived assuming steady-state operation.

From Table 4 it can be seen that the theoretically predicted and the experimentally obtained squared estimation errors exhibit good agreement. If the number of random test cases were increased to a suitably large number, it is expected that the theoretical and experimental results would be identical. It can also be seen that all three estimators are able to produce unbiased estimates of the combustor exit temperature, T40; however, their estimates of LPT exit temperature, T50, net thrust, Fn, and LPC stall margin, SmLPC, are biased. The encouraging finding is that the new systematic approach to tuner selection significantly reduces the overall mean squared estimation error compared to the other two approaches. Relative to the conventional approach of tuner selection the experimental mean squared estimation errors in T40, T50, Fn and SmLPC are reduced 76, 82, 80 and 63 percent, respectively. It can also be observed that the SVD tuner selection approach, which is designed to reduce the estimation error bias, does in fact reduce the sum of squared biases relative to the subset of health parameters approach. However, the SVD approach is also found to increase the estimation variance, which contributes to its overall mean squared estimation error.

TABLE 4.-AUXILIARY PARAMETER SQUARED ESTIMATION ERRORS

\begin{tabular}{|c|l|c|c|c|c|}
\hline Tuners & \multicolumn{1}{|c|}{ Error } & $\begin{array}{c}\mathrm{T} 40 \\
\left({ }^{\circ} \mathrm{R}\right)\end{array}$ & $\begin{array}{c}\mathrm{T} 50 \\
\left({ }^{\circ} \mathrm{R}\right)\end{array}$ & $\begin{array}{c}\text { Fn } \\
(\%)\end{array}$ & $\begin{array}{c}\text { SmLPC } \\
(\%)\end{array}$ \\
\hline \multirow{2}{*}{$\begin{array}{c}\text { Subset of } \\
\text { health } \\
\text { parameters }\end{array}$} & Theor. sqr. bias & 0.00 & 561.76 & 3.84 & 3.28 \\
& Theor. variance & 74.76 & 29.65 & 0.48 & 0.34 \\
& Theor. sqr. error & 74.76 & 591.41 & 4.31 & 3.62 \\
& Exper. sqr. error & 74.90 & 583.29 & 4.27 & 3.60 \\
\hline \multirow{3}{*}{ SVD tuner } & Theor. sqr. bias & 0.00 & 512.46 & 4.05 & 5.28 \\
selection & Theor. variance & 65.99 & 67.21 & 0.80 & 1.31 \\
& Theor. sqr. error & 65.99 & 579.67 & 4.86 & 6.59 \\
& Exper. sqr. error & 66.20 & 579.39 & 4.98 & 6.76 \\
\hline \multirow{2}{*}{ Systematic } & Theor. sqr. bias & 0.00 & 87.81 & 0.66 & 0.95 \\
tuner & Theor. variance & 17.49 & 18.55 & 0.13 & 0.35 \\
selection & Theor. sqr. error & 17.49 & 106.35 & 0.79 & 1.30 \\
& Exper. sqr. error & 17.61 & 106.54 & 0.86 & 1.35 \\
\hline
\end{tabular}

A visual illustration of the effect that tuner selection has on Kalman filter estimation accuracy can be seen in Figures 2 through 5, which show actual and estimated results for the auxiliary parameters T40, T50, Fn and SmLPC respectively. 
Each plot shows a $300 \mathrm{~s}$ segment of the evaluated test cases. The step changes that can be observed in each plot every $30 \mathrm{~s}$ correspond to a transition to a different health parameter vector. True model auxiliary parameter outputs are shown in black, and Kalman filter estimates are shown in red. In each figure the information is arranged top to bottom according to tuner selection based upon: a) a subset of health parameters; b) singular value decomposition; and c) the new systematic selection strategy. The information shown in these figures corroborates the information in Table 4; namely all three tuner selection approaches produce unbiased estimates of T40 (Figure 2), while the systematic tuner selection strategy yields a noticeable reduction in the total squared estimation error (squared bias plus variance) of all four auxiliary parameters.

\section{Comparison with Maximum a Posteriori Estimation}

The presented systematic tuner selection strategy minimizes the mean squared error of the on-line estimator at steady-state operating conditions, taking advantage of prior knowledge of engine health parameter distributions. As such it is somewhat analogous to the maximum a posteriori (MAP) estimation method which is commonly applied for ground-based aircraft gas turbine engine gas path analysis (Refs. 5 and 6). This leads to the question, how does the on-line Kalman filter estimation accuracy compare to MAP estimation accuracy? Prior to making this comparison the mathematical formulation of the MAP estimator is briefly introduced. Here a steady-state model of the measurement process in the following form is applied

$$
y_{k}=H h_{k}+v_{k}
$$

where the matrix $H$ relates the effects of the health parameter vector, $h$, to the sensed measurements, $y$. From Eq. (19), it can be seen that $H$ is equivalent to $C(I-A)^{-1} L+M$. The maximum a posteriori (MAP) estimator follows the closed form expression

$$
\hat{h}_{k}=\left(P_{h}^{-1}+H^{T} R^{-1} H\right)^{-1} H^{T} R^{-1} y_{k}
$$

The MAP estimator is capable of estimating more unknowns than available measurements due to the inclusion of a priori knowledge of the estimated parameter covariance, $P_{h}$. However, the MAP estimator, unlike a Kalman filter, is not a recursive estimator and does not take advantage of past measurements to enhance its estimate at the current time step. Furthermore, the MAP estimator only considers a static relationship between system state variables and measured outputs-it does not consider system dynamics. Because of these differences a Kalman estimator with optimally selected tuning parameters should outperform the MAP estimator. However, under steady-state conditions, with minimal sensor noise the two estimation approaches should produce similar results. To test this theory, a MAP estimator was designed and its estimation accuracy was compared to a Kalman filter with tuning parameters optimally selected to minimize the estimation errors in the health parameter vector $h$. First, the two estimators were designed and evaluated using the original sensor noise levels shown in Table 2. Next, the sensor noise levels were set to $1 / 20^{\text {th }}$ of their original levels, the estimators were re-designed, and the comparison was repeated. Monte Carlo simulation evaluations as previously described were applied (i.e., 375 random health parameter vectors, $30 \mathrm{~s}$ in duration, with estimation accuracy calculations based upon the last $10 \mathrm{~s}$ of each $30 \mathrm{~s}$ test case). Theoretical and experimental estimation errors are shown in Table 5 and Table 6 for the original noise and reduced noise levels, respectively. At original noise levels the Kalman estimator is able to produce smaller estimation errors. However, at the reduced noise level the two estimation approaches are found to be nearly identical. This comparison validates that the Kalman estimation approach is indeed producing a minimum mean squared estimation error as intended, while providing the capability to support real-time on-line estimation under dynamic operating scenarios.

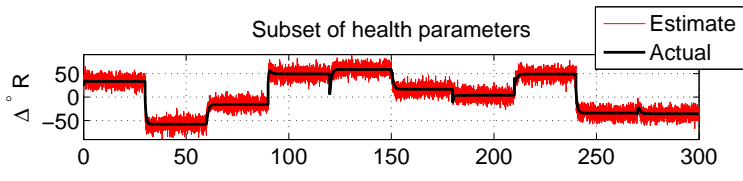

SVD tuner selection

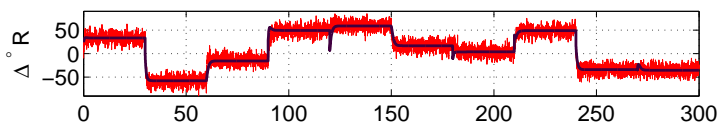

Systematic tuner selection

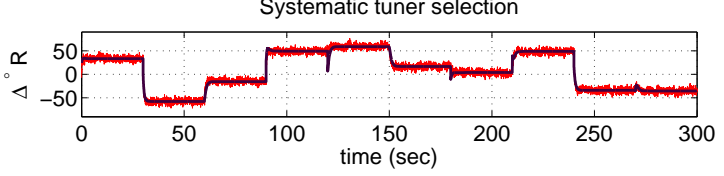

Figure 2.-T40 estimation (tuner comparison).
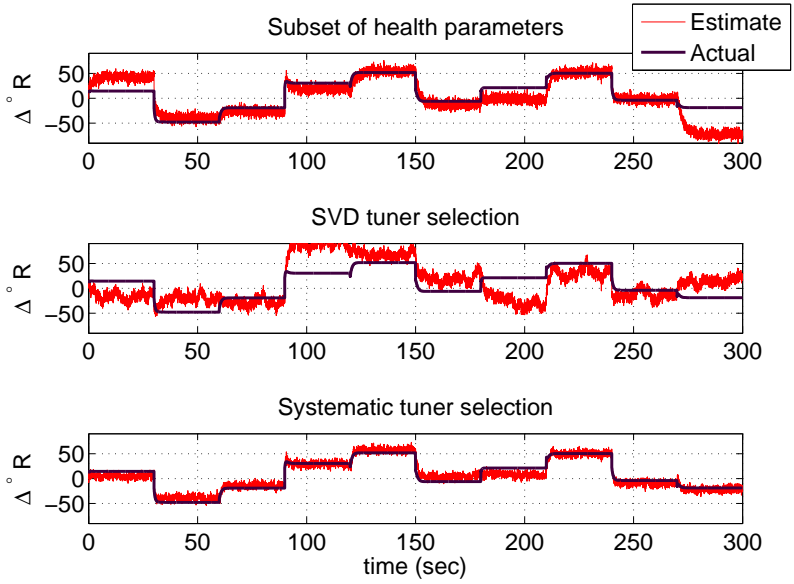

Figure 3.-T50 estimation (tuner comparison). 

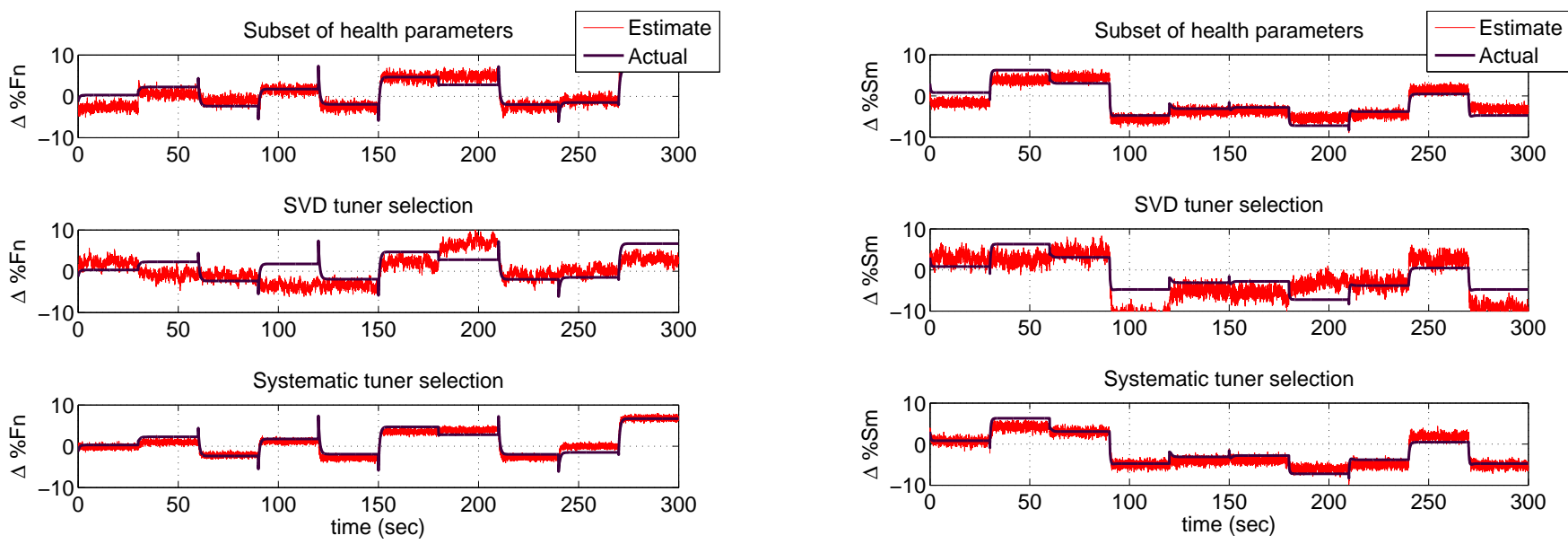

Figure 4.-Fn estimation (tuner comparison).

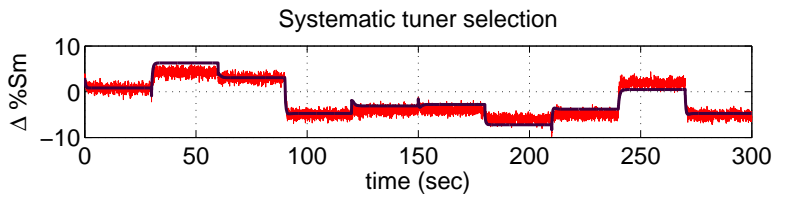

Figure 5.-SmLPC estimation (tuner comparison).

TABLE 5.-HEALTH PARAMETER PERCENT SQUARED ESTIMATION ERRORS (NOMINAL NOISE)

\begin{tabular}{|c|c|c|c|c|c|c|c|c|c|c|c|c|}
\hline Estimator & Error type & $h 1$ & $h 2$ & $h 3$ & $h 4$ & $h 5$ & h6 & $h 7$ & $h 8$ & h9 & $h 10$ & Sum \\
\hline \multirow{2}{*}{$\begin{array}{l}\text { Kalman } \\
\text { filter }\end{array}$} & Theor. squared error & 2.00 & 1.67 & 3.07 & 1.66 & 0.40 & 0.68 & 0.96 & 0.21 & 2.32 & 3.14 & 16.09 \\
\hline & Exper. squared error & 1.82 & 1.60 & 2.77 & 1.59 & 0.41 & 0.68 & 0.97 & 0.21 & 2.13 & 3.15 & 15.31 \\
\hline \multirow{4}{*}{$\begin{array}{c}\text { MAP } \\
\text { estimator }\end{array}$} & Theor. squared bias & 2.42 & 1.75 & 3.53 & 1.90 & 0.47 & 0.96 & 1.00 & 0.16 & 2.47 & 3.17 & 17.81 \\
\hline & Theor. variance & 0.22 & 0.27 & 0.16 & 0.64 & 0.69 & 0.71 & 0.36 & 0.36 & 0.17 & 0.09 & 3.68 \\
\hline & Theor. squared error & 2.63 & 2.02 & 3.69 & 2.54 & 1.16 & 1.67 & 1.36 & 0.52 & 2.64 & 3.26 & 21.48 \\
\hline & Exper. squared error & 2.52 & 1.89 & 3.44 & 2.45 & 1.14 & 1.57 & 1.34 & 0.51 & 2.49 & 3.24 & 20.60 \\
\hline
\end{tabular}

TABLE 6.-HEALTH PARAMETER PERCENT SQUARED ESTIMATION ERRORS (REDUCED NOISE)

\begin{tabular}{|c|c|c|c|c|c|c|c|c|c|c|c|c|}
\hline Estimator & Error type & $h 1$ & $h 2$ & $h 3$ & $h 4$ & $h 5$ & h6 & $h 7$ & $h 8$ & h9 & $h 10$ & Sum \\
\hline $\begin{array}{c}\text { Kalman } \\
\text { filter }\end{array}$ & Theor. squared error & 1.29 & 1.48 & 2.30 & 1.22 & 0.01 & 0.02 & 0.74 & 0.00 & 1.98 & 3.06 & 12.10 \\
\hline \multirow{4}{*}{$\begin{array}{c}\text { MAP } \\
\text { estimator }\end{array}$} & Theor. squared bias & 1.26 & 1.48 & 2.28 & 1.22 & 0.00 & 0.00 & 0.74 & 0.00 & 1.97 & 3.06 & 12.00 \\
\hline & Theor. variance & 0.03 & 0.00 & 0.04 & 0.01 & 0.01 & 0.02 & 0.00 & 0.00 & 0.01 & 0.00 & 0.13 \\
\hline & Theor. squared error & 1.30 & 1.48 & 2.32 & 1.22 & 0.01 & 0.02 & 0.74 & 0.00 & 1.99 & 3.06 & 12.14 \\
\hline & Exper. squared error & 1.14 & 1.41 & 1.98 & 1.17 & 0.01 & 0.02 & 0.75 & 0.00 & 1.79 & 3.08 & 11.34 \\
\hline
\end{tabular}

\section{Discussion}

While the systematic tuner selection approach presented here appears promising for on-line Kalman filter based parameter estimation applications, there are several practical considerations which need to be assessed when applying such a technique. The optimization routine attempts to minimize the overall squared estimation error-both bias and varianceunder steady-state operating conditions. The minimization of the estimation variance in particular can come at the expense of dynamic responsiveness of the Kalman filter. To illustrate this consider the time history plots of actual versus estimated T40 shown in Figure 6. The top plot shows Kalman filter estimation results using a tuning parameter vector systematically selected to minimize the error in four auxiliary parameters (T40, T50, Fn and SmLPC) as presented in the previous section. The bottom plot shows Kalman filter estimation results using a tuning parameter vector systematically selected to minimize the estimation error in T40 only. At time $100 \mathrm{~s}$ a step change in the health parameter input vector is introduced into the engine model; this allows the dynamic response of the two estimators to be compared. It can be observed that T40 estimation variance in the bottom plot is reduced, as is the mean steady-state estimation error ( $>300 \mathrm{~s})$. This is not surprising since one would generally expect improved results when optimizing to minimize the error in a single parameter, as opposed to multiple parameters. However, the estimator shown in the 

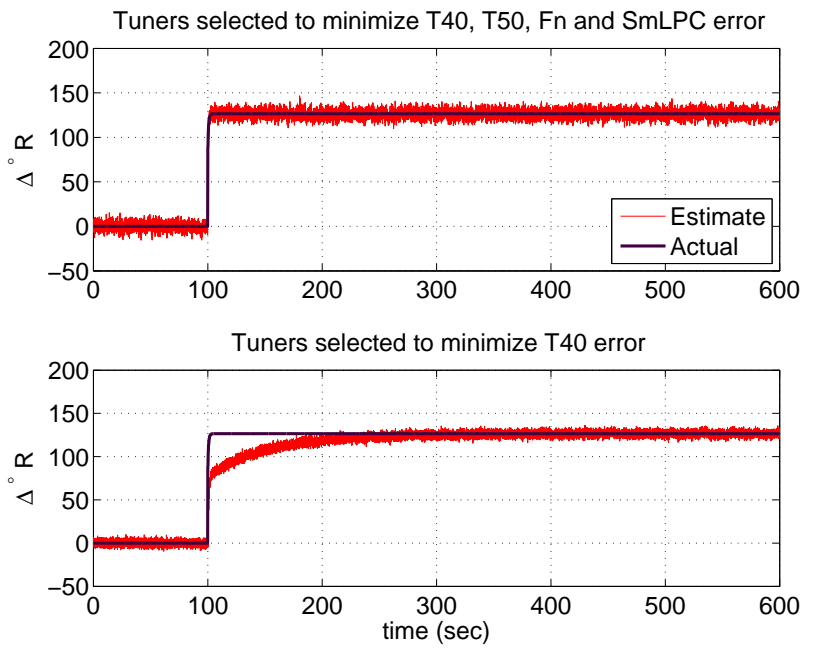

Figure 6.-Illustration of tuner impact on estimator response.

bottom plot does require a significantly longer time to reach steady-state convergence. Conversely, the estimator designed to minimize the steady-state error in four auxiliary parameters (top plot) is unable to place as much emphasis on T40 estimation variance reduction, but it is able to track dynamic changes in T40 more rapidly. This example illustrates the inter-dependence between estimation variance and responsiveness. Therefore, it is prudent for a designer to evaluate the Kalman filter to ensure that it tracks engine dynamics acceptably. If the dynamic response is unacceptable, the optimization routine can be rerun placing more weight on estimation error bias reduction, and less weight on variance reduction.

All results presented in this paper are based on a turbofan engine linear state-space model at a single operating point. While this linear assessment enables experimental validation of theoretical predictions, it is not representative of actual aircraft gas turbine engine operation which is a transient nonlinear system operating over a broad range of operating conditions. In order for the presented approach to be applicable it would need to be able to produce an optimal set of tuning parameters, not just at a single operating point but rather a globally optimal tuning parameter vector universally applicable over the range of operating conditions that an engine is expected to experience. A potential approach to selecting a single "globally optimal" tuning parameter vector is to modify the optimization routine to minimize the combined estimation error over multiple engine operating points such as takeoff, climb and cruise. This would be a straightforward modification to the Matlab optimization routine, but it would increase the computational time required to calculate the result. Since the systematic tuner selection process is only envisioned to be done once during the system design process, this will not impact the on-line execution speed of the Kalman filter. It is anticipated that the application of globally optimal tuners will result in some estimation accuracy degradation relative to tuners optimized for individual operating points, although this has not yet been verified or quantified.

\section{Conclusions}

A systematic approach to tuning parameter selection for online Kalman filter based parameter estimation has been presented. This technique is specifically applicable for the underdetermined aircraft engine parameter estimation case where there are fewer sensor measurements than unknown health parameters which will impact engine outputs. It creates and applies a linear transformation matrix, $V^{*}$, to select a vector of tuning parameters which are a linear combination of all health parameters. The tuning parameter vector is selected to be of low-enough dimension to be estimated, while minimizing the mean-squared error of Kalman filter estimates. The multi-parameter iterative search routine applied to optimally select $V^{*}$ was presented. Results have shown that while the transformation matrix returned by the optimization routine is not unique (different matrices can be found which produce a global minimum of the objective function), the routine is effective in returning a transformation matrix which is optimal, or near optimal, regardless of its initial starting guess of the matrix. The efficacy of the systematic approach to tuning parameter selection was demonstrated by applying it to parameter estimation in an aircraft turbofan engine linear point model. It was found to significantly reduce mean squared estimation errors compared to the conventional approach of selecting a subset of health parameters to serve as tuners. In some parameters the mean squared estimation error reduction was found to be over 80 percent. These estimation improvements were theoretically predicted and experimentally validated through Monte Carlo simulation studies.

The systematic approach to Kalman filter design is envisioned to be applicable for a broad range of on-board aircraft engine model-based applications which produce estimates of unmeasured parameters. This includes modelbased controls, model-based diagnostics, and on-board life usage algorithms. It is also envisioned to have benefits for sensor selection during the engine design process, specifically for assessing the performance estimation accuracy benefits of different candidate sensor suites. Areas for future work include extending the technique to produce a tuning parameter vector optimal over a range of operating conditions, and evaluating the technique on a non-linear engine model, under both steady-state and transient operating conditions.

\section{References}

1. Luppold, R.H. Roman, J.R., Gallops, G.W., Kerr, L.J., (1989), "Estimating In-Flight Engine Performance Varaiations Using Kalman Filter Concepts," AIAA-89-2584, AIAA 25 ${ }^{\text {th }}$ Joint Propulsion Conference. 
2. Volponi, A., (2008), "Enhanced Self-Tuning On-Board RealTime Model (eSTORM) for Aircraft Engine Performance Health Tracking," NASA/CR-2008-215272.

3. Kumar, A., Viassolo, D., (2008), "Model-Based Fault Tolerant Control," NASA/CR-2008-215273.

4. Litt, J.S., (2008), "An Optimal Orthogonal Decomposition Method for Kalman Filter-Based Turbofan Engine Thrust Estimation," Journal of Engineering for Gas Turbines and Power, Vol. 130/011601-1.

5. Doel, D.L., (1994), "An Assessment of Weighted-LeastSquares-Based Gas Path Analysis," J. of Engineering for Gas Turbines and Power, Vol. 116, pp. 336-373.
6. Volponi, A.J., et al, (2003), "Gas Turbine Condition Monitoring and Fault Diagnostics", Von Karman Institute for Fluid Dynamics, Lecture Series 2003-01.

7. España, M.D., (1994), "Sensor Biases Effect on the Estimation Algorithm for Performance-Seeking Controllers," J. Propulsion and Power, 10, pp. 527-532.

8. Simon, D., (2006), "Optimal State Estimation, Kalman, $\mathrm{H}_{\infty}$, and Nonlinear Approaches," John Wiley \& Sons, Inc., Hoboken, NJ.

9. Frederick, D.K., DeCastro, J.A., Litt, J.S., (2007), “User's Guide for the Commercial Modular Aero-Propulsion System Simulation (C-MAPSS)," NASA/TM-2007-215026. 


\begin{tabular}{|c|c|c|}
\hline \multicolumn{2}{|c|}{ REPORT DOCUMENTATION PAGE } & $\begin{array}{l}\text { Form Approved } \\
\text { OMB No. 0704-0188 }\end{array}$ \\
\hline \multicolumn{3}{|c|}{ 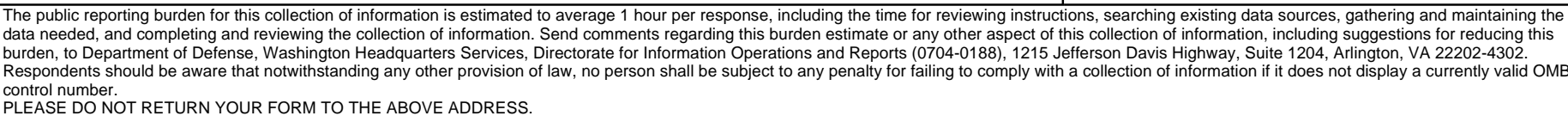 } \\
\hline $\begin{array}{l}\text { 1. REPORT DATE (DD-MM-YYYY) } \\
01-01-2010\end{array}$ & $\begin{array}{l}\text { 2. REPORT TYPE } \\
\text { Technical Memorandum }\end{array}$ & 3. DATES COVERED (From - To) \\
\hline \multirow{3}{*}{\multicolumn{2}{|c|}{$\begin{array}{l}\text { 4. TITLE AND SUBTITLE } \\
\text { Optimal Tuner Selection for Kalman Filter-Based Aircraft Engine }\end{array}$}} & 5a. CONTRACT NUMBER \\
\hline & & 5b. GRANT NUMBER \\
\hline & & 5c. PROGRAM ELEMENT NUMBER \\
\hline \multirow{3}{*}{\multicolumn{2}{|c|}{$\begin{array}{l}\text { 6. AUTHOR(S) } \\
\text { Simon, Donald, L.; Garg, Sanjay }\end{array}$}} & 5d. PROJECT NUMBER \\
\hline & & 5e. TASK NUMBER \\
\hline & & $\begin{array}{l}\text { 5f. WORK UNIT NUMBER } \\
\text { WBS 645846.02.07.03.03.012 }\end{array}$ \\
\hline \multicolumn{2}{|c|}{$\begin{array}{l}\text { 7. PERFORMING ORGANIZATION NAME(S) AND ADDRESS(ES) } \\
\text { National Aeronautics and Space Administration } \\
\text { John H. Glenn Research Center at Lewis Field } \\
\text { Cleveland, Ohio 44135-3191 }\end{array}$} & $\begin{array}{l}\text { 8. PERFORMING ORGANIZATION } \\
\text { REPORT NUMBER } \\
\text { E-17111 }\end{array}$ \\
\hline \multirow{2}{*}{\multicolumn{2}{|c|}{$\begin{array}{l}\text { 9. SPONSORING/MONITORING AGENCY NAME(S) AND ADDRESS(ES) } \\
\text { National Aeronautics and Space Administration } \\
\text { Washington, DC 20546-0001 }\end{array}$}} & $\begin{array}{l}\text { 10. SPONSORING/MONITOR'S } \\
\text { ACRONYM(S) } \\
\text { NASA }\end{array}$ \\
\hline & & $\begin{array}{l}\text { 11. SPONSORING/MONITORING } \\
\text { REPORT NUMBER } \\
\text { NASA/TM-2010-216076; GT2009-59684 }\end{array}$ \\
\hline \multicolumn{3}{|c|}{$\begin{array}{l}\text { 12. DISTRIBUTIONIAVAILABILITY STATEMENT } \\
\text { Unclassified-Unlimited } \\
\text { Subject Category: } 07 \\
\text { Available electronically at http://gltrs.grc.nasa.gov } \\
\text { This publication is available from the NASA Center for AeroSpace Information, 443-757-5802 }\end{array}$} \\
\hline
\end{tabular}

\section{SUPPLEMENTARY NOTES}

\section{ABSTRACT}

A linear point design methodology for minimizing the error in on-line Kalman filter-based aircraft engine performance estimation applications is presented. This technique specifically addresses the underdetermined estimation problem, where there are more unknown parameters than available sensor measurements. A systematic approach is applied to produce a model tuning parameter vector of appropriate dimension to enable estimation by a Kalman filter, while minimizing the estimation error in the parameters of interest. Tuning parameter selection is performed using a multi-variable iterative search routine which seeks to minimize the theoretical mean-squared estimation error. This paper derives theoretical Kalman filter estimation error bias and variance values at steady-state operating conditions, and presents the tuner selection routine applied to minimize these values. Results from the application of the technique to an aircraft engine simulation are presented and compared to the conventional approach of tuner selection. Experimental simulation results are found to be in agreement with theoretical predictions. The new methodology is shown to yield a significant improvement in on-line engine performance estimation accuracy.

\section{SUBJECT TERMS}

Aircraft engines; Systems health monitoring; Gas turbine engines; Kalman filtering; State estimation

\begin{tabular}{|c|c|c|c|c|c|}
\hline \multicolumn{3}{|c|}{ 16. SECURITY CLASSIFICATION OF: } & \multirow{2}{*}{$\begin{array}{l}\text { 17. LIMITATION OF } \\
\text { ABSTRACT } \\
\text { UU }\end{array}$} & \multirow{2}{*}{$\begin{array}{l}\text { 18. NUMBER } \\
\text { OF } \\
\text { PAGES } \\
19\end{array}$} & \multirow{2}{*}{$\begin{array}{l}\text { 19a. NAME OF RESPONSIBLE PERSON } \\
\text { STI Help Desk (email:help@sti.nasa.gov) } \\
\text { 19b. TELEPHONE NUMBER (include area code) } \\
\text { 443-757-5802 }\end{array}$} \\
\hline $\begin{array}{l}\text { a. REPORT } \\
\text { U }\end{array}$ & $\begin{array}{l}\text { b. ABSTRACT } \\
\text { U }\end{array}$ & $\begin{array}{l}\text { c. THIS } \\
\text { PAGE } \\
\text { U }\end{array}$ & & & \\
\hline
\end{tabular}



